\title{
Fourier Coefficients of OGLE Variables
}

\author{
Siobahn Morgan, Melanie Simet, Stephanie Bargenquast, Chris \\ Dickerson \\ Department of Earth Science, University of Northern Iowa, Cedar Falls \\ IA, 50614-0335, USA
}

\begin{abstract}
Fourier coefficients for the variable stars in the OGLE survey of the Galactic Center and several globular clusters are presented. The characteristics of the RR Lyrae, $\delta$ Scuti and SX Phe stars are presented and a comparison to those observed in other studies is made. In some cases the coefficients of the stars can be used to determine distances and the physical characteristics of the variables.
\end{abstract}

The Fourier coefficients for over 300 stars observed by the OGLE (Udalski et al., 1994-1997) project were obtained and used to derive characteristics of RR Lyrae stars as well as distance determinations to the Galactic bulge and towards various clusters. These results were previously published elsewhere (Morgan, Simet, \& Bargenquast 1998a,b,c; Morgan \& Dickerson 1999) and will not be given in detail here. Amongst the results is the finding that physical characteristics (mass, luminosity and effective temperature) for RR Lyrae stars found in the Galactic bulge and $\omega$ Cen are similar to those found in previous studies, with most RR Lyrae stars having a metallicity near -1.1 for the Galactic bulge, and near -1.5 for $\omega$ Cen. The distance to the Galactic bulge based upon the RR Lyrae stars is $m-M \approx 14.5$, while for $\omega$ Cen a distance modulus of $m-M=13.74$ is found. The entire text of the poster paper as well as the previously published results and data can be found at the WWW address http://ww. earth.uni.edu/OGLE/.

\section{References}

Morgan, S. M., Simet, M., \& Bargenquast, S. 1998a, Acta Astron., 48, 331

Morgan, S. M., Simet, M., \& Bargenquast, S. 1998b, Acta Astron., 48, 341

Morgan, S. M., Simet, M., \& Bargenquast, S. 1998c, Acta Astron., 48, 509

Morgan, S. M. \& Dickerson, C. 1999, submitted to Acta Astron.

Udalski, A., Kubiak, M., Szymański, M., et al. 1994, Acta Astron., 44, 317

Udalski, A., Szymański, M., Kałużny, J., et al. 1995a, Acta Astron., 45, 1

Udalski, A., Olech, A., Szymański, M., et al. 1995b, Acta Astron., 45, 433

Udalski, A., Olech, A., Szymański, M., et al. 1996, Acta Astron., 46, 51

Udalski, A., Olech, A., Szymański, M., et al. 1997, Acta Astron., 47, 1 